\title{
Расчет энергетической структуры точечных дефектов в нитриде алюминия методами теории функционала плотности
}

\author{
Александров И.А., Журавлев К.С. \\ ИФП СО РАН, 630090, Новосибирск, пр. Ак. Лаврентьева, 13
}

DOI 10.34077/Semicond2019-314

Определение энергетической структуры и оптических свойств точечных дефектов в кристаллах является фундаментальной задачей, решение которой необходимо для улучшения качества материалов, используемых для создания оптических и электронных приборов. Нитриды металлов третьей группы перспективны для создания светодиодов и лазерных диодов ультрафиолетового и видимого диапазона, мощных высокочастотных транзисторов, ультрафиолетовых фотоприемников, источников одиночных фотонов, работающих при комнатной температуре. Для идентификации полос фотолюминесценции и поглощения в нитриде алюминия в данной работе проведены расчеты энергий формирования, уровней термодинамических переходов и формы полос фотолюминесценции и поглощения точечных дефектов в AlN методами теории функционала плотности. Расчеты проводились с использованием гибридного функционала HSE [1] и оптимизированных сохраняющих норму псевдопотенциалов Вандербита [2] в программном пакете Quantum Espresso [3]. Расчетная ширина запрещенной зоны соответствует экспериментальной Eg=6.1 эВ [4] при значении параметра $\alpha$ гибридного функционала HSE, отвечающего за долю короткодействующего Хартри-Фоковского обменного функционала, равном 0.33. Расчеты энергетической структуры дефектов проводились с энергией отсечки при разложении на плоские волны $\mathrm{E}_{\text {cut }}=80 \mathrm{Ry}$, с использованием 96-атомной сверхъячейки и смещенной относительно начала координат сетки Монкхорста-Пака $2 \times 2 \times 2$ для интегрирования по зоне Бриллюэна. При расчете энергетической структуры заряженных дефектов проводилась корректировка, учитывающая взаимодействие зарядов в периодически повторяющейся сверхъячейке [5]. Результат расчета параметров решетки и энтальпии формирования объемного AlN $\mathrm{a}=3.11 \AA, \mathrm{c}=4.97 \AA, \Delta \mathrm{H}_{\mathrm{f}}=3.15$ эВ хорошо согласуется с экспериментальными данными $\mathrm{a}=3.11 \AA$, $\mathrm{c}=4.98 \AA, \Delta \mathrm{H}_{\mathrm{f}}=3.28$ эВ [6,7]. Расчет формы полос фотолюминесценции проводился с использованием метода, предложенного работе [8]. Согласно расчетам, термодинамические уровни переходов между зарядовыми состояниями (-3/-2), (-2/-1), (-1/0) и (0/+1) вакансии алюминия в AlN расположены на 3.16 эВ, 2.91 эВ, 2.49 эВ и 1.34 эВ выше дна зоны проводимости. Термодинамический уровень перехода (-1/0) примеси углерода в подрешетке азота $\mathrm{C}_{\mathrm{N}}$ расположен на 1.99 эВ выше дна зоны проводимости. Примесь кислорода в подрешетке азота $\mathrm{O}_{\mathrm{N}}$ проявляет свойства DX-центров, для которых присоединение второго электрона к нейтральному дефекту приводит к сильной релаксации решетки с образованием глубокого уровня. В положительно заряженном и нейтральном состоянии $\mathrm{O}_{\mathrm{N}}$ наиболее энергетически выгодным является положение кислорода в узле решетки на месте азота. В отрицательно заряженном состоянии наиболее энергетически выгодным является положение кислорода в смещенном относительно гексагональной оси положении, кроме того, локальный минимум энергии наблюдается при смещении атома кислорода вдоль гексагональной оси в тетраэдрическое междоузлие.

Работа поддержана Российским научным фондом (проект 18-72-00136).

[1] J. Heyd, G. E. Scuseria, and M. Ernzerhof, J. Chem. Phys. 124, 219906 (2006).

[2] D. R. Hamann, Phys. Rev. B 88, 085117 (2013).

[3] P. Giannozzi et al., J. Phys.:Condens. Matter 29465901 (2017).

[4] M. Feneberg et al., Phys. Rev. B 82, 075208 (2010).

[5] C. Freysoldt, J. Neugebauer, and C. G. Van de Walle, Phys. Rev. Lett. 102, 016402 (2009).

[6]I. Vurgaftman, J. R. Meyer, Journal of Applied Physics 94, 3675 (2003).

[7] M. Ranade et al., J. Mater. Res., 16(10), 2824(2001).

[8] A. Alkauskas, J. L. Lyons, D. Steiauf, and C. G. Van de Walle. Phys. Rev. Lett 109, 267401 (2012). 